\title{
ADAPTATION ACCURACY OF TWO DIFFERENT DENTURE BASE MATERIALS FOR THE COMPLETELY EDENTULOUS MAXILLARY ARCH: AN IN VITRO STUDY
}

\author{
Nagla Nassouhy *
}

\begin{abstract}
Objectives: to compare between adaptation accuracy of metallic denture base and injectionmolded denture base for the edentulous maxillary arch.

Materials and Methods: Ten stone casts were obtained from duplicating an educational model of a maxillary edentulous arch. The casts were divided into two equal groups; group I received metallic denture bases while group II received denture bases constructed from injection-molded Thermopress. The dentures and their respective casts were sectioned and gap measurements were made in the mid-palatal and crestal areas using a travelling microscope.
\end{abstract}

Results: gap measurements at the mid-palatal areas were significantly higher than those at the crestal areas in both groups. On comparing the two groups, there was no significant difference between the two groups both at the crestal and mid-palatal areas. Conclusions: Metal denture bases and injection molded dentures base show comparable levels of adaptation accuracies and are both suitable alternatives for heat cured PMMA denture bases.

\section{INTRODUCTION}

Conventional heat cured polymethyl methacrylate (PMMA) is the dominant material for fabricating complete dentures due to its simple processing technique and its ability to provide acceptable biological, physical and esthetic results at a reasonable $\operatorname{cost}^{1-3}$. Compression molding of heat cured PMMA is a well established technique for denture processing ${ }^{4}$. Two unavoidable problems with this technique are polymerization shrinkage and the release of thermal stresses which inevitably have an effect on the adaptation accuracy of the final denture ${ }^{5,6}$. The presence of intimate contact and accurate fit of the denture base to the underlying mucosa is essential for achieving retention and stability, especially that complete dentures rely almost entirely on physical means for retention $^{7,8}$. Heat cured PMMA also suffers from some mechanical and physical problems such as low impact and fatigue strengths as well as low thermal conductivity and hardness. Furthermore,

\footnotetext{
* Lecturer of Removable Prosthodontics, Cairo University
} 
the presence of residual monomer causes tissue irritation and porosities resulting in denture hygiene and esthetic problems in the long run ${ }^{9,10}$.

Alternative materials and techniques are available to overcome the shortcomings of heat cured PMMA, including metal alloys and thermoplastic resins. Metal alloys, like cobalt chromium, offer superior mechanical and physical properties in comparison with PMMA. They are stronger, with higher fatigue and fracture strengths ${ }^{1}$.In addition, they can be cast in thin sections without compromising their rigidity or fracture resistance. This property, in addition to their high thermal conductivity, provides patients with a natural "feel" to the prosthesis. This reduced palatal thickness also decreases interference with phonation and allows stimulation of the underlying mucosa ${ }^{7}$. Metallic denture bases are also biocompatible, dimensionally stable with high surface polish which facilitates hygiene maintenance ${ }^{11}$. They are particularly useful in situations with high risk of fracture that require strengthening of the denture base, as in cases with bruxism or in single maxillary dentures $^{12,13}$. Problems with metallic denture base include esthetics and difficulty to perform rebasing procedures $^{1}$.

Injection molding techniques using thermoplastic resins have gained popularity in recent years as they are free from residual monomer and porosities. In addition, polymerization shrinkage is compensated by the continuous injection of the resin at a certain pressure $^{14}$. They also have high impact strength and resistance to cracking in addition to their superior esthetics. Several studies have reported on the improved adaptation accuracy of injection molding techniques when compared to conventional heat cured processing ${ }^{14-18}$. However, injection molding is an expensive technique that requires special laboratory equipment as well as skilled technicians ${ }^{19}$.
A number of methods are available for evaluation of the adaptation accuracy of denture bases ${ }^{20}$. Commonly used methods include weighing an elastomeric material impressed beneath the denture base $^{6}$, image analysis methods ${ }^{6}$, profile projectors ${ }^{21}$ and microscopic measurements ${ }^{18}$. The travelling microscope has an accuracy of $0.001 \mathrm{~mm}$ and a magnification of 50 times making it highly accurate for gap measurements ${ }^{22}$.

Several materials and techniques are now available for the fabrication of complete denture bases that aim at improving denture base esthetics, physical and mechanical properties as well as accuracy of adaptation. Metal denture bases are known for the superior adaptation properties ${ }^{7}$, but the literature comparing their adaptation accuracy to resin denture bases seems to be lacking. This study was conducted to compare between the adaptation accuracy of metallic denture base and that of injection molded denture base for the maxillary edentulous arch.

\section{MATERIALS AND METHODS}

For accurate measurement of adaptation of the denture bases, the casts used in this study had to be similar in shape and size in order to fabricate uniform denture bases. An educational model of a maxillary edentulous arch with a moderately curved palatal vault and no undercuts was duplicated into ten stone casts. This was done to exclude the effect of different palatal vault shapes or undercuts on the adaptation of the denture bases. A silicon* mold was fabricated for the model and the model was duplicated ten times using type IV dental stone. The ten master casts were divided into two equal groups; Group I receiving a maxillary denture with a metallic denture base, and Group II receiving an acrylic resin denture constructed by

\footnotetext{
* Replisil 22n, Germany
} 
injection molding technique. Adaptation accuracy was measured for the two groups using a travelling microscope.

\section{Group I}

The casts were duplicated into refractory casts for fabrication of the metal denture base. Prefabricated plastic patterns were adapted to the refractory casts to create a pattern for the metal base. The pattern was designed to completely cover the palatal region and the residual ridge with meshwork saddles for mechanical retention of the acrylic resin and teeth to the metal base ${ }^{23}$. Patterns were then sprued with an inverted sprue, invested and casted in cobalt chromium alloy* using conventional techniques. The metal framework was returned to the master cast and checked for proper seating and fit. Then, one layer of base plate wax $1.5 \mathrm{~mm}$ in thickness was adapted to the remaining parts of the denture base and the buccal and labial flanges of the denture. Artificial teeth were arranged as it was reported that the presence of denture teeth has an effect on the adaptation accuracy of denture bases ${ }^{24}$. The trial denture base was waxed up, and then denture was flasked and processed in heat cured acrylic resin ${ }^{* *}$ in the conventional manner. The dentures were finished and polished.

\section{Group II}

One layer of base plate wax of $1.5 \mathrm{~mm}$ thickness was adapted to the casts. Holes were drilled in the ridge lap area of the artificial teeth for mechanical bonding before they were arranged as injectionmolded denture base does not bond chemically with acrylic resin teeth. A silicon mold of the waxed-up trial denture base was made to duplicate the occlusal and polished surfaces to provide uniform thickness and tooth positioning for the remaining denture bases as recommended by Parvizi et al ${ }^{15}$. Denture bases were flasked according to the manufacturer's instructions and processed by injection molding in thermoplastic injectable resin ${ }^{* * *}$ using Thermopress injection molding unit ${ }^{* * * *}$.

After deflasking of the dentures, they were left to bench cool to room temperature. Then, excess material was trimmed and the dentures were polished with a wet rag and pumice while keeping them on their respective casts to avoid any distortion before measurement of the adaptation accuracy.

\section{Measurements}

A transverse section was made in each cast with its respective denture extending $5 \mathrm{~mm}$ anterior to the posterior border of the denture. Sectioning was done using a vertical trimmer with a diamond cutting disc under water cooling as recommended by Lee et $\mathrm{al}^{6}$. Measurements were done at 3 locations; mid-palataly and crestaly on each side (fig 1,2). For standardization purposes, the locations were marked on one cast using an indelible pencil then they were transferred to the rest of the casts by means of an acrylic resin template with vertical slots opposite to the markings.

Measurements were done using a travelling microscope $^{* * * * *}($ fig $3,4,5)$. This microscope has an accuracy of $0.001 \mathrm{~mm}$ and a magnification of $50 \mathrm{x}$ making it highly accurate for gap measurements ${ }^{22}$. Each gap at each location was measured 3 times then a mean of the measurements for each location was obtained. The measurements were tabulated for statistical analysis.

\footnotetext{
* Cobalt-chromium metal framework, Veta, Switzerland.

** Vertex regular, Zeist, Netherlands

*** Polyan IC, Modefied methacrylate, Bredent, Germany

**** Thermopress 400 version 2.4/2.56, Bredent, Germany

****** Carlzeis, Jenna, Germany
} 


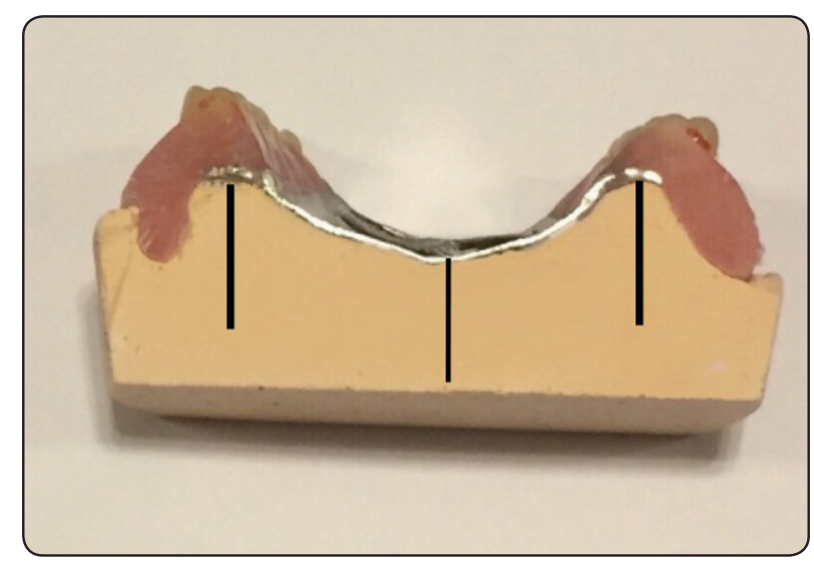

Fig. (1) The sectioned metallic denture base on the cast

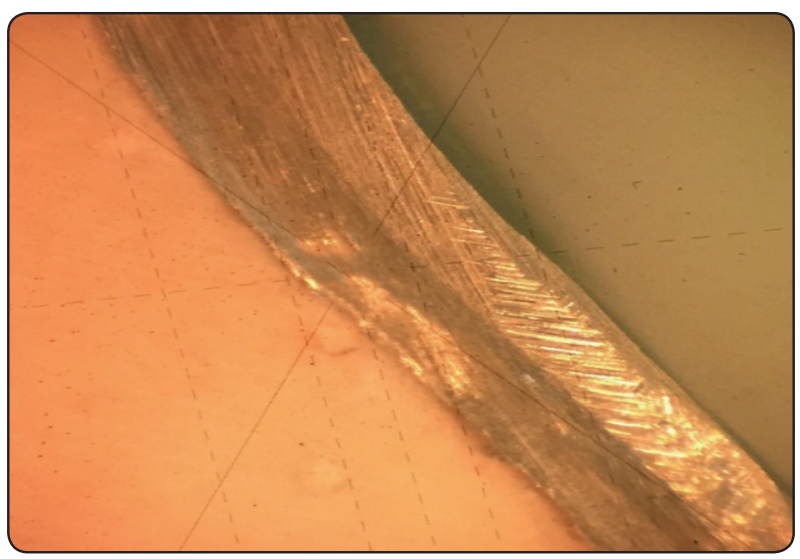

Fig. (3) Microscopic image of the metal denture base

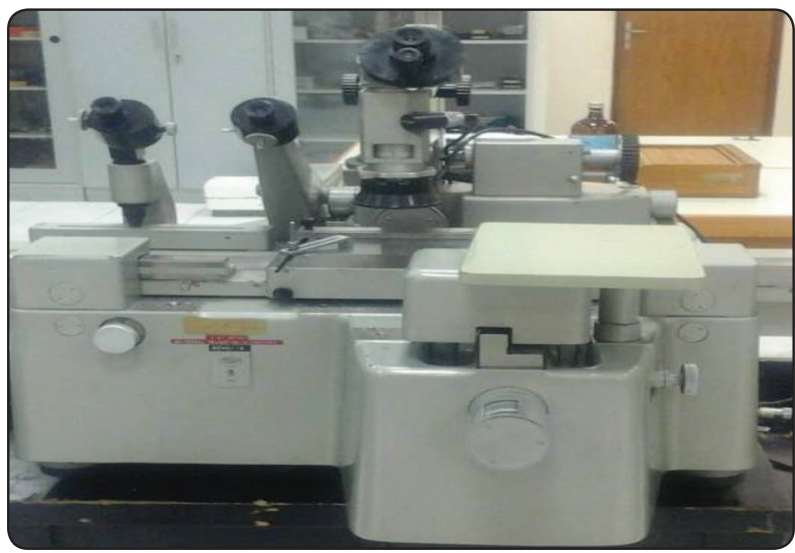

Fig. (5) The travelling microscope used for gap measurements

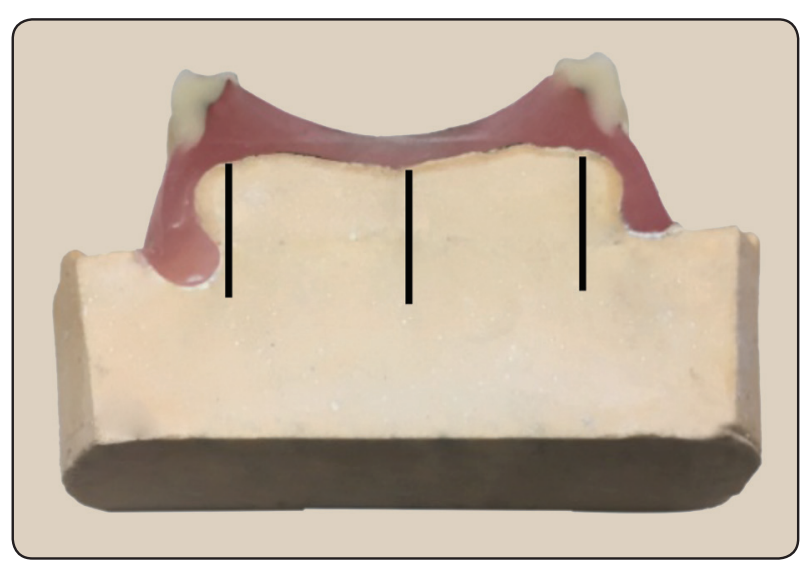

Fig. (2) The sectioned injection molded denture base on the cast

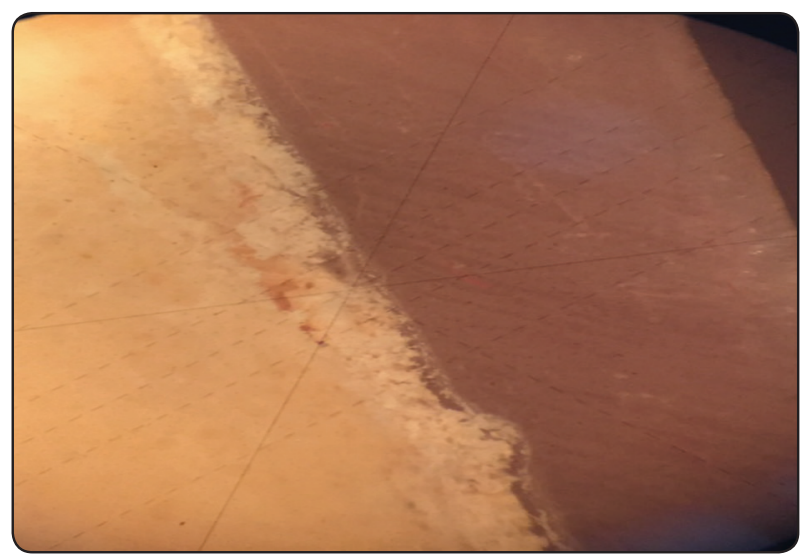

Fig. (4) Microscopic image of the injection-molded denture base

\section{Statistical Analysis}

The mean and standard deviation values were calculated for each group. Data were explored for normality using Kolmogorov-Smirnov and Shapiro-Wilk tests and showed parametric (normal) distribution. One way ANOVA was used to compare between independent samples in more than two groups. Independent sample-t test was used to compare between independent samples between two groups. The significance level was set at $\mathrm{P} \leq$ 0.05 . Statistical analysis was performed with IBM ${ }^{\circledR}$ SPSS ${ }^{\circledR}$ Statistics Version 20 for Windows. 


\section{RESULTS}

The results of the mid-palatal and crestal measurements for each group are shown in Table 1. There was no significant difference between the two crestal gap measurements on either side of the arch in the two groups. Therefore, a mean of the two sides was obtained and used for comparisons (table 1, fig 6). On comparing the mid-palatal and crestal measurements, a statistically significant difference was found in both groups. In Group I, the highest gap measurement was found mid-palatally $(0.410 \pm 0.001 \mathrm{~mm})$ while the lowest measurement was found crestally $(0.307 \pm 0.002 \mathrm{~mm})$. As for group II, the highest gap measurement was also found mid-palatally $(0.403 \pm 0.006 \mathrm{~mm})$ while the least mean value was found in crestally $(0.302 \pm$ $0.005 \mathrm{~mm}$ ). On comparing the mid-palatal section of the two groups, no significant difference was found $(\mathrm{p}=0.121)$. There was also no significant difference between the two groups at the crestal sections $(\mathrm{p}=0.053)($ table 2 , fig 6,7).

TABLE (1) The mean, standard deviation (SD) values of gap measurements in all sections of both groups

\begin{tabular}{|c|c|c|c|c|}
\hline \multirow{2}{*}{ Variables } & \multicolumn{4}{|c|}{ Gap Measurements } \\
\cline { 2 - 5 } & \multicolumn{2}{|c|}{ Group I } & \multicolumn{2}{c|}{ Group II } \\
\cline { 2 - 5 } & Mean & SD & Mean & SD \\
\hline Mid-palatal & $0.410^{\mathrm{a}}$ & 0.001 & $0.403^{\mathrm{a}}$ & 0.006 \\
\hline Crestal right & $0.307^{\mathrm{b}}$ & 0.003 & $0.303^{\mathrm{b}}$ & 0.006 \\
\hline Crestal left & $0.306^{\mathrm{b}}$ & 0.002 & $0.301^{\mathrm{b}}$ & 0.004 \\
\hline $\boldsymbol{P}$-value & $\mathbf{5 0 . 0 0 1 *}$ & $\mathbf{\leq 0 . 0 0 1} *$ \\
\hline
\end{tabular}

Means with different small letters in the same column indicate statistically significance difference, *; significant $(p<0.05) \quad n s ;$ non-significant $(p>0.05)$

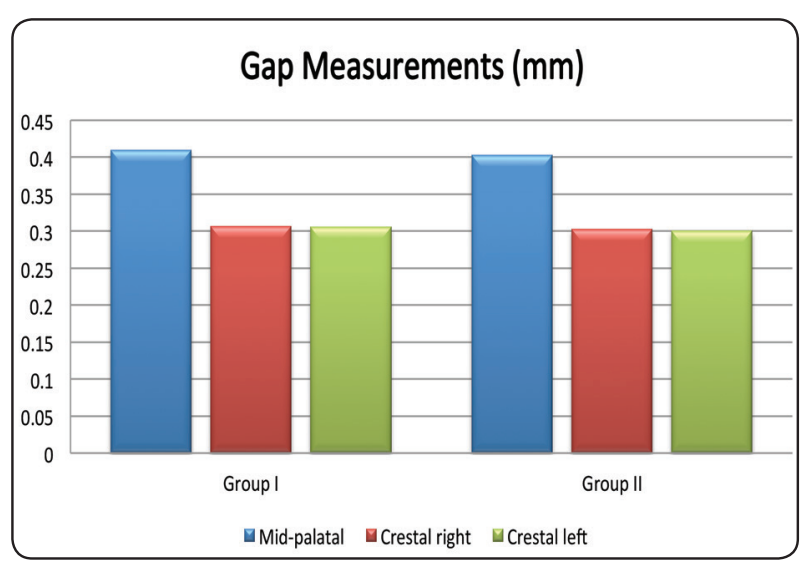

Fig. (6) Bar chart showing mean gap measurements in all sections in both groups

TABLE (2): The mean, standard deviation (SD) values of mid-palatal and crestal gap measurements in both groups

\begin{tabular}{|c|c|c|c|c|c|}
\hline \multirow{3}{*}{ Variables } & \multicolumn{5}{|c|}{ Gap Measurements } \\
\hline & \multicolumn{2}{|c|}{ Group I } & \multicolumn{2}{|c|}{ Group II } & \multirow{2}{*}{ P-value } \\
\hline & Mean & SD & Mean & SD & \\
\hline Mid-palatal & $0.410^{\mathrm{aA}}$ & 0.001 & $0.403^{\mathrm{aA}}$ & 0.006 & $0.121 n s$ \\
\hline Crestal & $0.307^{\mathrm{bA}}$ & 0.002 & $0.302^{\mathrm{bA}}$ & 0.005 & $0.053 n s$ \\
\hline P-value & \multicolumn{2}{|c|}{$\leq 0.001 *$} & \multicolumn{2}{|c|}{$\leq 0.001 *$} & \\
\hline
\end{tabular}

Means with different small letters in the same column indicate statistically significance difference, means with different capital letters in the same row indicate statistically significance difference. *; significant $(p<0.05) \quad n s ;$ non-significant $(p>0.05)$

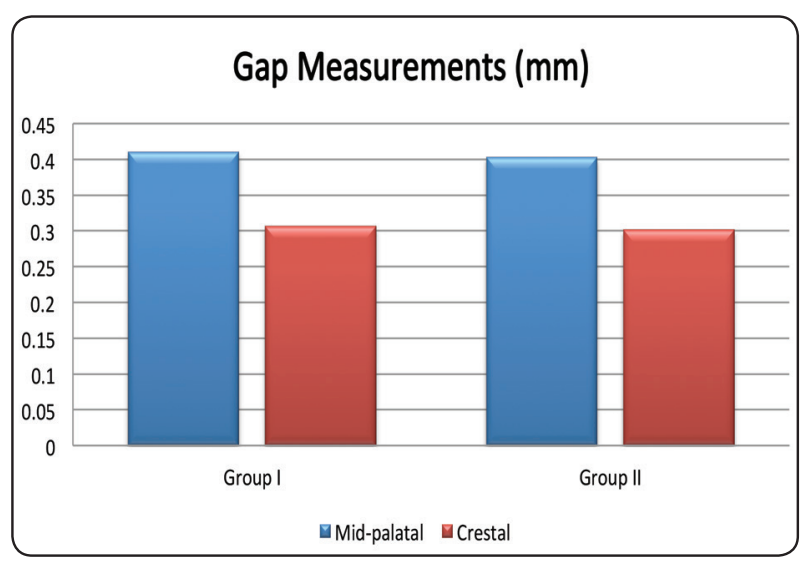

Fig. (7) Bar chart showing comparison of mean mid-palatal and crestal gap measurements in both groups 


\section{DISCUSSION}

The complete denture depends primarily on physical means to achieve sufficient retention. This is attained through intimate contact and adaptation of the denture base to the underlying mucosa ${ }^{8}$. Denture base material is one of the factors that affect the adaptation and accuracy of fit of the final denture. Improving the fit of the denture could decrease the initial adaptation period needed by the complete denture patient.

The results of this study showed that mid-palatal sections had significantly higher gap measurements than crestal sections in both groups, which comes in accordance with previous studies ${ }^{6,25}$. Parvizi et al ${ }^{15}$ reported that the processing technique had a great influence on the dimensional changes that occur during the processing procedure. It is important to note that shrinkage that occurs during solidification of the material (whether metal or injection molded resin) is compensated to a great extent by the feeding of the additional material in the sprue channels. Furthermore, the use of an inverted sprue directed the shrinkage of the denture base towards the cast. These minor changes, in addition to the thermal contraction of the solid material offer an explanation for the dimensional changes that resulted in gap formation. These changes are considered linear rather than volumetric, occurring towards the center of the denture. The significantly higher gap measurements mid-palatally more than crestally could be due to the greater bulk of the denture base material in that area.

On comparing the two groups, there was no difference in gap measurements at the mid-palatal areas. There was also no difference between the crestal gap measurements of the two groups. Overall, the two groups demonstrated comparable adaptation accuracies at both mid-palatal and crestal areas.

There are several materials available in the market nowadays for the fabrication of denture bases. It is not possible for one material to achieve all the ideal requirements of a denture base. Each material is bound to have advantages as well as drawbacks, rendering it particularly useful in some clinical situations and unsuitable in others. The strength, rigidity, biocompatibility, thermal conductivity and dimensional stability of the metal denture base come at the expense of esthetics and inability to rebase the denture later on. On the other hand, the advantages of the injection molded material such as high impact strength and lack of porosities come at the expense of higher cost and technique sensitivity. The results of this study show that both investigated materials show comparable levels of adaptation accuracies rendering them good alternatives for conventional heat cured PMMA depending on the requirements of the presented clinical situation. Finally, more studies are needed to determine the effect of adaptation accuracy on the clinical performance of the final denture, and whether it is important for the long term success of the denture or just to obtain a stable retentive denture in the early stages of use.

\section{CONCLUSIONS}

Within the limitations of this study, metallic denture base and injection molded denture base show comparable levels of adaptation accuracies and are both suitable alternatives for heat cured PMMA denture bases.

\section{REFERENCES}

1. Anusavice KJ. Phillips' science of dental materials. 11th ed. St. Louis: Saunders Co, 2003.

2. Takamata T, Setcos JC. Resin denture bases: review of accuracy and methods of polymerization. Int J Prosthodont 1989; 2: 555-62.

3. Memon MS, Yunus N, Razak a a. Some mechanical properties of a highly cross-linked, microwave-polymerized, injection-molded denture base polymer. Int J Prosthodont 2001; 14: 214-218.

4. Phoenix RD, Mansueto MA, Ackerman NA, et al. Evaluation of mechanical and thermal properties of commonly used denture base resins. J Prosthodont 2004; 13: 17-27. 
5. Garfunkel E. Evaluation of dimensional changes in complete dentures processed by injection-pressing and the pack-and-press technique. J Prosthet Dent 1983; 50: 757-76.

6. Lee C-J, Bok S-B, Bae J-Y, et al. Comparative adaptation accuracy of acrylic denture bases evaluated by two different methods. Dent Mater J 2010; 29: 411-417.

7. Rahn AO HC. Text Book of Complete Dentures. Lea and Febiger: Philadelphia (PA), 1993.

8. Darvell BW, Clark RKF. The physical mechanisms of complete denture retention. Br Dent J 2000; 189: 248-252.

9. Eichhold WA WJ. Denture base acrylic resins: friend or foe? Compendium 1990; 11: 720-5.

10. Phoenix RD. Denture base materials. Dent Clin North Am 1996; 40: 113-20.

11. Perezous LF, Stevenson GC, Flaitz CM, et al. The effect of complete dentures with a metal palate on Candida species growth in HIV-infected patients. J Prosthodont 2006; 15: 306-315.

12. Darbar UR, Huggett R, Harrison A. Denture fracture--a survey. Br Dent J 1994; 176: 342-5.

13. Chopra D, Gupta V, Sethi P. Maxillary Complete Denture with Metal Palate Base : A Case Report Corresponding Address : Dr . Devendra Chopra. J Dent Sci oral Rehabil 2013; Jan-March: 44-46.

14. Ono T, Kita S, Nokubi T. Dimensional accuracy of acrylic resin maxillary denture base polymerized by a new injection pressing method. Dent Mater J 2004; 23: 348-52.

15. Parvizi A, Lindquist T, Schneider R, et al. Comparison of the dimensional accuracy of injection-molded denture base materials to that of conventional pressure-pack acrylic resin. J Prosthodont 2004; 13: 83-89.

16. Ganzarolli SM, De Mello JAN, Shinkai RS, et al. Internal adaptation and some physical properties of methacrylatebased denture base resins polymerized by different techniques. J Biomed Mater Res - Part B Appl Biomater 2007; 82: 169-173.

17. Gharechahi J,Asadzadeh N,Shahabian F, et al. Dimensional changes of acrylic resin denture bases: conventional versus injection-molding technique. J Dent (Tehran) 2014; 11: 398-405.

18. Moussa, A.R. Zaki D.Y. Gabry H AT. Comparative Adaptation Accuracy of Heat Cured and Injection Molded Resin DentureBase Materials. J Appl Sci Res 2012; 8: 4691-4696.

19. Shah J, Bulbule N, Kulkarni S, et al. Comparative evaluation of sorption, solubility and microhardness of heat cure polymethylmethacrylate denture base resin \& flexible (thermoplastic polyamide nylon) denture base resin. J Clin Diagnostic Res; 8. Epub ahead of print 2014. DOI: $10.7860 / J C D R / 2014 / 8707.4770$.

20. Zissis A, Huggett R, Harrison A. Measurement methods used for the determination of dimensional accuracy and stability of denture base materials. Journal of Dentistry 1991; 19: 199-206.

21. Pavan S, Arioli Filho JN, Dos Santos PH, et al. Effect of microwave treatments on dimensional accuracy of maxillary acrylic resin denture base. Braz Dent J 2005; 16: 119-123.

22. Shawky Y YH. Adaptation accuracy and retention of injection - and compression - molded maxillary complete denture: in - vitro and in - vivo study. EDJ 2014; 1011-7.

23. Postic S. Design of complete denture reinforced with metal base. Stomatol Glas Srb 2013; 60: 15-23.

24. Baemmert RJ, Lang BR, Barco MT, et al. Effects of denture teeth on the dimensional accuracy of acrylic resin denture bases. Int J Prosthodont 1990; 3: 528-37.

25. Consani RLX, Domitti SS, Consani S. Effect of a new tension system, used in acrylic resin flasking, on the dimensional stability of denture bases. J Prosthet Dent 2002; 88: 285-289. 